\title{
Analytical imaging studies of the migration of degraded orpiment, realgar, and emerald green pigments in historic paintings and related conservation issues
}

\author{
Katrien Keune ${ }^{1,2^{*}}$, Jennifer Mass ${ }^{3}$, Apurva Mehta ${ }^{4}$, Jonathan Church $^{5}$ and Florian Meirer ${ }^{6}$
}

\begin{abstract}
Yellow orpiment $\left(\mathrm{As}_{2} \mathrm{~S}_{3}\right)$ and red-orange realgar $\left(\mathrm{As}_{4} \mathrm{~S}_{4}\right)$ photo-degrade and the nineteenth-century pigment emerald green $\left(\mathrm{Cu}\left(\mathrm{C}_{2} \mathrm{H}_{3} \mathrm{O}_{2}\right)_{2} \cdot 3 \mathrm{Cu}\left(\mathrm{AsO}_{2}\right)_{2}\right)$ degrades into arsenic oxides. Because of their solubility in water, arsenic oxides readily migrate and are found throughout the multi-layered paint system. The widespread arsenic migration has consequences for conservation, and this paper provides better insight into the extent of the problem. Five paint samples containing orpiment, realgar or emerald green pigments deriving from paintings by De Heem (17th C), Van Gogh (19th C), Rousseau (19th C), an unknown 17th C northern European artist and an Austrian painted cupboard (19th C) were investigated using SEM/EDX, imaging ATR-FTIR and arsenic (As) K-edge $\mu$-XANES to obtain the spatial distribution and chemical speciation of arsenic in the paint system. In all of the samples investigated arsenic had migrated throughout the multi-layered paint structure of the art object, from support to varnish. Furthermore, $\mathrm{As}^{5+}$-species were found throughout the entire paint sample. We hypothesize that arsenic trioxide is first formed, dissolves in water, further oxidizes to arsenic pentaoxide, and then reacts with lead, calcium and other ions and is deposited in the paint system as insoluble arsenates. Since the degradation of arsenic pigments such as orpiment, realgar and emerald green occurs through a highly mobile intermediate stage, it not only affects the regions rich in arsenic pigments, but also the entire object, including substrate and top varnish layers. Because of this widespread potential for damage, preventing degradation of arsenic pigments should be prioritized and conservators should minimize exposure of objects containing arsenic pigments to strong light, large fluctuations in relative humidity and water-based cleaning agents.
\end{abstract}

Keywords: Orpiment, Realgar, Emerald green, Migration, Painting, Arsenic, Degradation, XANES, Imaging

\section{Background}

Yellow orpiment $\left(\mathrm{As}_{2} \mathrm{~S}_{3}\right)$ and red-orange realgar $\left(\mathrm{As}_{4} \mathrm{~S}_{4}\right)$, used by artists from different cultures since antiquity, and emerald green $\left(\mathrm{Cu}\left(\mathrm{C}_{2} \mathrm{H}_{3} \mathrm{O}_{2}\right)_{2} \cdot 3 \mathrm{Cu}\left(\mathrm{AsO}_{2}\right)_{2}\right)$, a popular brilliant green pigment first synthesized by Willem Sattler in 1814, are pigments that contain arsenic. They have all been found to be unstable in paints. Photo-degradation of realgar $\left(\mathrm{As}_{4} \mathrm{~S}_{4}\right)$ and orpiment $\left(\mathrm{As}_{2} \mathrm{~S}_{3}\right)$ results in

\footnotetext{
${ }^{*}$ Correspondence: k.keune@rijksmuseum.nl

${ }^{2}$ Van't Hoff Institute for Molecular Sciences, University of Amsterdam,

Science Park 904, 1098, XH, Amsterdam, The Netherlands

Full list of author information is available at the end of the article
}

sensitive, transparent, whitish, friable and/or crumbling paints, while chemically degraded emerald green pigment $\left(\mathrm{Cu}\left(\mathrm{C}_{2} \mathrm{H}_{3} \mathrm{O}_{2}\right)_{2} \cdot 3 \mathrm{Cu}\left(\mathrm{AsO}_{2}\right)_{2}\right.$, copper (II) acetoarsenite) in oil paint leads to transparent, dark brown layers [1] or stains on paper [2]. The degradation of these arsenic-containing pigments seriously affects the appearance and stability of paintings and painted objects, the chemical changes are irreversible, and the accompanying physical changes to the rest of the object worsen the conservation problems. The physical alteration associated with the chemical degradation, often seen as cracking and crumbling of paint layers and surfaces, further enhances the degradation and poses a major conservation challenge [3, 4]. The cracked 
surfaces easily collect dirt from the environment, accentuating the discolored regions, and are vulnerable to further degradation when exposed to moisture and solvents during treatment. While discolored and crumbling paint can be consolidated, it cannot be readily cleaned. The open, porous structure creates an unvarnished painting (or painted furniture)-like surface. Re-varnishing the affected regions of the work, unfortunately, does not mitigate the problem because the highly mobile degradation products migrate through the varnish. The previous study indicated that the photo-degradation products of orpiment and realgar are highly water soluble, and consequently they do not remain at the location of the original pigment in the paint, but migrate throughout the whole multi-layered paint system [5]. For example, the investigation of an early eighteenth-century chest on stand that had been repeatedly varnished after the application of an orpiment design showed that the photo-oxidized arsenic species had migrated through each varnish layer, with larger deposits visible at the interface of each pair of layers [5].

Migrated and dispersed products containing arsenic also form a potential health hazard during conservation practice. The conservator might not be aware that arsenic is likely to be present in, and at the surface of, varnish layers further away from the degraded arsenic-containing paint layers. In the case of paintings, conservation practice often involves the removal and replacement of discolored varnishes. In addition, when original pigment material has migrated into these varnishes, and their removal will expose friable altered paint while not improving the color of the altered pigment underneath, it may actually be prudent to consider leaving the varnish intact in these discolored regions. In the case of painted American furniture, conservation practice typically consists of leaving the full finish history of the object intact, unless the figure of the wood or the painted decoration is completely obscured. In this case the topmost layers of finish will be mechanically reduced by careful sanding, which again leads to a health hazard with arsenic species having migrated into these uppermost varnish layers. Like many photo-oxidation problems observed in artists' pigments, often the original brightly colored paint can be observed below the discolored and flaking altered paint. This has led to the undesirable consequence of folk/vernacular art objects exhibiting this type of alteration to be "skinned" by untrained restorers, removing the artists' original but damaged material to reveal the bright colors underneath. While such a restoration presents the owner or potential buyer with an attractive presentation surface, not only has original artist's material been removed, but the pigments thus exposed lose the protection of the overlying alteration crust and will most likely experience rapid degradation.
The degradation of realgar and orpiment is triggered by light within the wavelength range of $500-670 \mathrm{~nm}$. The highest degradation efficiency is in the green part of the visible light spectrum $(530-560 \mathrm{~nm})$ [6]. In the first step of light induced degradation, realgar undergoes polymorphism and becomes friable and bright yellow, $\left(\mathrm{As}_{\mathrm{x}} \mathrm{S}_{\mathrm{y}}\right.$, para-realgar) and subsequently degrades further to a white phase $\left(\mathrm{As}_{2} \mathrm{O}_{3}\right.$, arsenolite). The photo-oxidation of orpiment, on the other hand, results directly in a white product (arsenolite), often appearing as a dirty/off white color or an ocherous material because of the presence of an overlying varnish $[6,7]$. In objects painted with large fields of orpiment, the degradation is often not uniform, resulting in a mix of ivory to ochre yellow [8]. The degradation products of arsenic sulfide pigments have been observed and identified in painted works of art by visual observation, $\mu$ Raman spectroscopy, and X-ray diffraction [9-18]. One limitation of using $\mu$ Raman spectroscopy to study realgar is the danger of creating para-realgar during the analysis due to the pigment's extreme photo-sensitivity. So far, para-realgar and arsenic trioxide have been consistently identified as the main oxidation products of arsenic sulfide pigments. Recent synchrotron radiationbased arsenic (As) K-edge micro X-ray absorption near edge structure ( $\mu$-XANES) studies following preliminary $\mu$ FTIR studies revealed another set of degradation products, i.e. arsenate compounds $\left(\mathrm{As}^{5+}\right)$ present as calcium, lead, aluminum and iron arsenates depending on the pigments and fillers surrounding the original arsenic sulfide pigments [5]. The arsenates $\left(\mathrm{As}^{5+}\right)$ are the phases that spread throughout the entire multi-layered paint system and dominate the photo-degraded paint, ground, and varnish layers, while the arsenite compounds $\left(\mathrm{As}^{3+}\right)$ seem to be located close to the intact arsenic sulfide pigment. We hypothesized that arsenic trioxide has to be oxidized in its aqueous form $\left(\mathrm{As}(\mathrm{OH})_{3}\right)$ to convert into arsenate phases. It is well known that arsenates are transported in the environment as an oxide via water [19]. So the presence of $\mathrm{As}^{5+}$ compounds in the paint systems indicates that the arsenic trioxide is dissolved by ambient water present in the paint and transported by water throughout the paint system. While the deleterious health effects of, for example, wallpapering rooms in damp climates with arsenical papers has been recognized since the nineteenth century, ours is the first thorough speciation and mobility study to be conducted for these pigments (see, for example, refs. [20-22]).

At the end of the nineteenth century artists were aware that emerald green should not be mixed with sulfurcontaining pigments such as yellow cadmium sulfide, as this leads to the formation of brown copper sulfides [23]. Nevertheless, emerald green is also found to degrade in the absence of sulfur-containing pigments leading to 
transparent discolored brown layers [1]. In this extreme case, the paint containing emerald green is medium rich with a relatively high concentration of monoacids, diacids and resin acids. These acids groups react with the emerald green pigment, forming copper carboxylates, like copper soaps $\left(4 \mathrm{Cu}(\mathrm{FA})_{2}\right)$ (FA $=$ fatty acids) and arsenic trioxide $\left(\mathrm{As}_{2} \mathrm{O}_{3}\right)$. The degradation mechanism of emerald green has been revealed by analytical imaging studies on paint samples from The Descent of the Cattle in the High Jura Mountains (1836) by Théodore Rousseau in the Mesdag Collection in The Hague, supported by chemical laboratory experiments [1]. The transparent, brown appearance is ascribed to the loss of the emerald green pigments as light reflectors in combination with darkening of the multiple thick medium-rich paint and varnish layers. Energy dispersive X-ray analyses indicate that, not unlike paintings containing realgar and/or orpiment, arsenic degradation products are found throughout a degrading painting containing emerald green in layer interfaces, varnishes and regions surrounding particles containing iron and/or aluminum. However, the chemical speciation of the arsenic-based reaction products in paintings containing emerald green has not yet been done, so the degradation mechanism is still not understood. In this paper we report arsenic (As) K-edge micro $\mathrm{X}$-ray absorption near edge structure ( $\mu$-XANES) analyses that have been carried out to identify the chemical speciation of the degradation products of emerald green containing arsenic.

To provide better insight into the extent of arsenic migration in paints, this paper will present elemental analyses, imaging ATR-FTIR, and synchrotron XANES studies of various historical paint samples. The samples containing realgar, orpiment and emerald green we selected were taken from seventeenth-century paintings by De Heem and an unknown northern seventeenthcentury artist, a nineteenth-century Austrian cupboard, and nineteenth-century paintings by Rousseau and Van Gogh. This information should help conservators to estimate the risks involved during display, handling, and conservation treatments.

\section{Results and discussion}

Localization of arsenic in degraded paints containing orpiment, realgar and emerald green

\section{Case study: The Dentist, seventeenth century, unknown}

\section{artist-realgar/orpiment}

The Dentist (private collection) is an oil painting on panel painted in the seventeenth century by an unknown artist (Fig. 1a). The pictorial image of The Dentist is based on Lucas van Leyden's engraving The Dentist (1523, Rijksmuseum, Amsterdam). A paint sample was taken from the orange skirt of the woman at the right. The paint sample is build up of wood from the panel (layer 0), a chalk-glue ground (layer 1), a transparent brown umber or Kassel earth-pigmented brown second layer (layer 2), followed by a lead white and carbon black pigmented underdrawing layer (layer 3) (Fig. 1b, c). Two paint layers were applied-a red layer pigmented with vermilion and red lake (chalk substrate) (layer 4), an orange layer with a mixture of realgar/orpiment and gypsum (present as an extender or substrate for a lake) where the upper part of the layer is photo-degraded (layer 5). The elemental spot analyses presented in Fig. 1d and e illustrate the presence of arsenic in all paint layers. Even in the wood structure from the panel support very low intensities of arsenic were detected (Fig. 1e). Yet no arsenic was detected with $\mathrm{X}$-ray elemental analysis [not shown] in another paint sample, taken from blue underskirt of the woman at the right. The arsenic released from the orpiment/realgar pigment migrated throughout the multi-layered paint system into the wood-structure of the panel.

\section{Case study: nineteenth-century cupboard-emerald green}

Emerald green was used as green paint in the varnished and highly decorated cupboard "Hl. Theresa" from the master of the Losensteinleitner Werkstätten (1824), Freilichtmuseum Sumerauerhof, St. Florian, Austria. The paint sample taken from the pilaster in the right upper corner comprises a primary layer containing white clay followed by green paint consisting of a mixture of emerald green, barium sulfate and lead white. The irregular, crystalline emerald green particles (20 microns) seem to still be intact and tightly packed in the compacted paint layer (Fig. 2). Nevertheless, arsenic was detected by elemental analysis in the emerald green particles as well as in varnish layer covering the emerald green layer (Fig. 2). Since emerald green is the only source of arsenic in this paint, arsenic detected in the varnish layer must be derived from the emerald green particles. Despite the fact that the emerald green paint on the cupboard seems to be intact, a relatively small quantity of emerald green has reacted away and arsenic is present in the vanish layer. This type of finding may have been previously attributed to the use of arsenic-containing pesticides in the first half of the twentieth century, but is now shown to be a result of pigment alteration.

\section{Case study: Van Gogh, nineteenth century-emerald green} Many of the green brush strokes in Interior of a Restaurant (1887), Vincent van Gogh, Kröller-Müller Museum, Netherlands (KM110.328/F342/JH 1256), became lighter resulting in green paint of heterogeneous color (Fig. 3a). The green paint contains emerald green and theoretically emerald green degrades into the white degradation product arsenic trioxide. Nevertheless, light microscope 

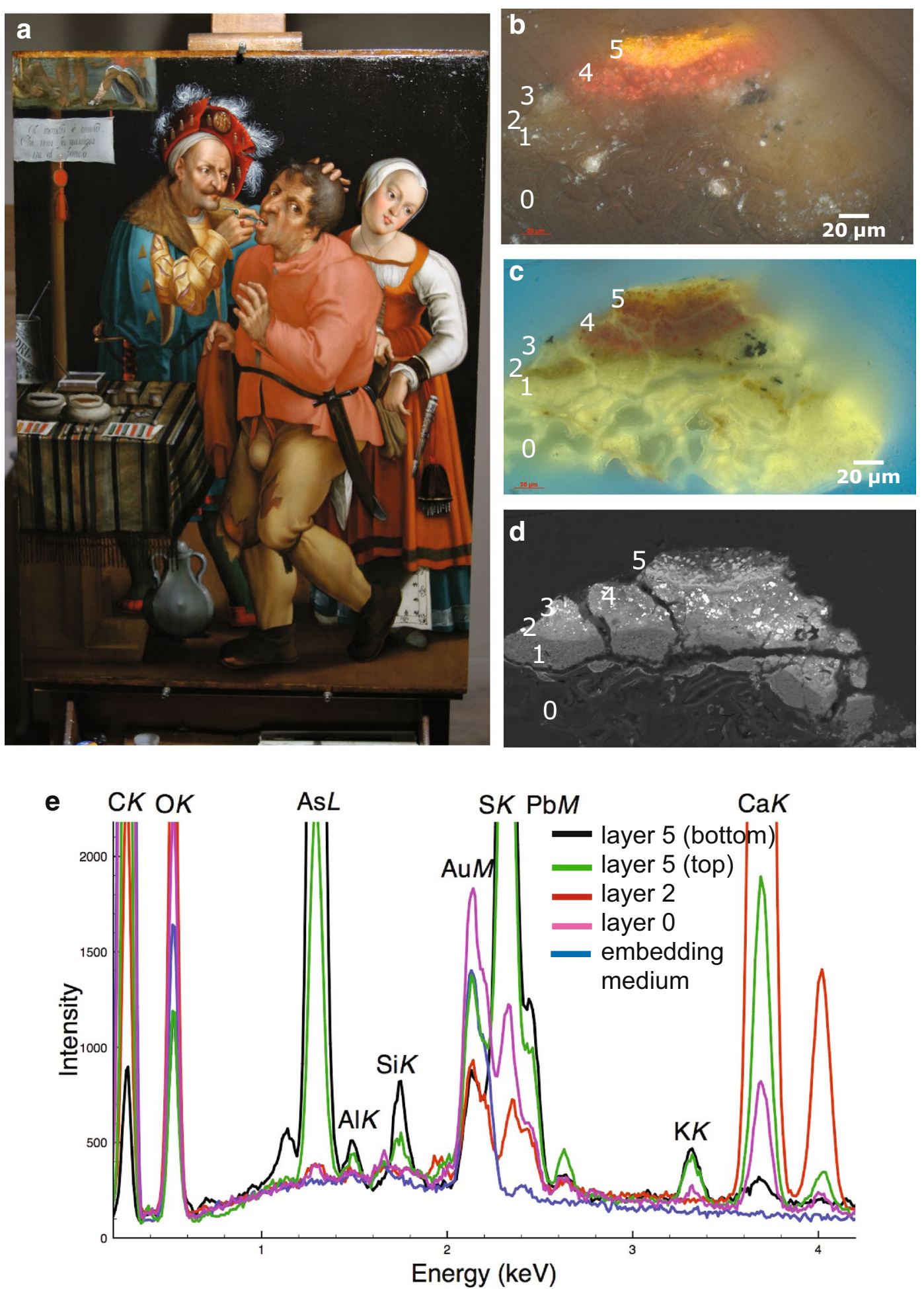

Fig. 1 Painting The Dentist (a), light microscopic image of paint sample taken from the orange skirt, normal light (b) and UV (c); backscatter electron image (d) and part of EDX spectrum (e). The black line represents spot analyses in lower part of layer 5 and the green line in the upper part of layer 5 , the realgar/orpiment pigmented layer; the red line represents spot analyses in layer 2, the under drawing; the magenta line in wood structure of the panel (layer 0); the blue line in the resin of the embedding medium 

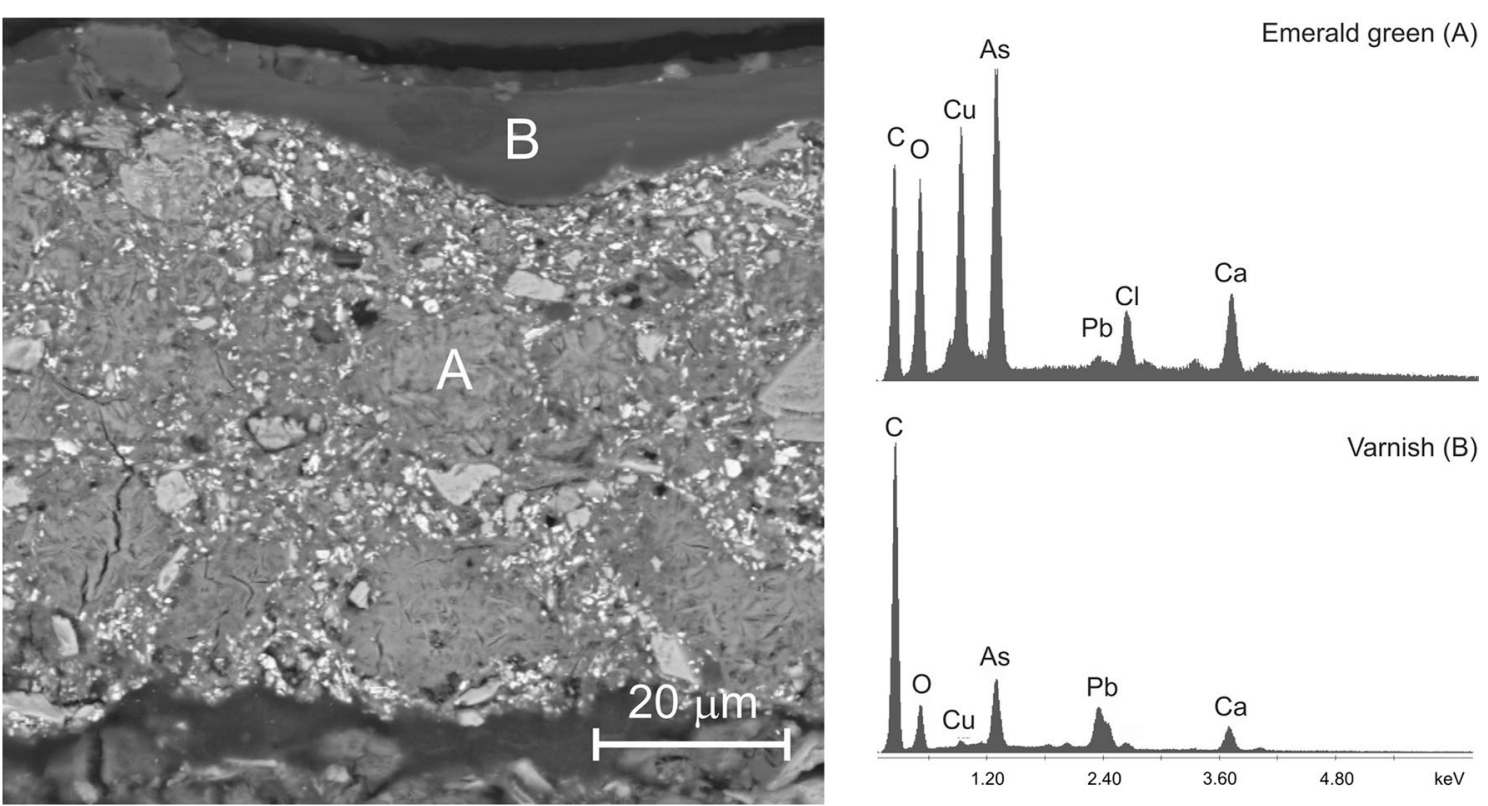

Fig. 2 Cupboard "HI. Theresa" from the master of the Losensteinleitner Werkstätten (1824) Freilichtmuseum Sumerauerhof, St. Florian, Austria. Backscatter electron image showing a detail of a paint cross-section taken from a green decoration in the cupboard "HI. Theresa" from the Losensteinleitner Werkstätten (left), elemental X-ray spectrum of emerald green particle (A) and varnish (B); Meister der Losensteinleitner (right) [27]

images and SEM/EDX analyses revealed an intact emerald green layer (Fig. 3b-h, sample structure is described in the figure caption). The irregular emerald green particles (1-10 microns) are densely packed with several fine lead white particles in between. Both pigments seem to have been unaffected by ageing as their particle edges appear sharp and well defined in the backscatter electron image (Fig. 3d). Figure 3i shows a line scan taken over the entire Van Gogh sample revealing the relative intensity of carbon, arsenic and lead throughout layers 1-5 including the embedding medium on both sides. High intensities of arsenic relate to the emerald green particles, and high lead intensities to lead white pigment in layer 5 (Fig. 3i). The line scan shows that arsenic is present throughout the entire lead white ground layer in low relative intensities. This indicates that although the paint layer containing emerald green seems to be intact, the pigment reacts with the acid-groups of the binder at the pigment-binder interface resulting a mobile arsenic degradation product.

The whitish appearance of the green paint is thus not caused by the emerald green. Large whitish, transparent masses were observed in layer 4 during polishing. A remnant of these masses is visible on the left edge of layer 4 (white arrow, Fig. 3b). This area is rich in zinc (Fig. 3h), while the rest of layer 4 has a low zinc intensity except for a few zinc hotspots that correlate with the spots that have the characteristic UV fluorescence of zinc oxide visible in the UV light image. Based on these observations, we hypothesize that zinc oxide has reacted away to form zinc soap aggregates. Zinc soap aggregates are suspected of causing the lightened appearance of the green paint and this phenomenon will be further investigated.

\section{Chemical speciation and localization of arsenic in degraded paints containing orpiment, realgar and emerald green \\ Case study: De Heem, seventeenth century-realgar/ orpiment}

The orpiment-containing paint in the citron of the Festoon of Fruits and Flowers (1660-1670), Jan Davidsz. de Heem, Rijksmuseum Amsterdam (SK-A-138), is photodegraded (Fig. 4). Currently the citron has a brownish appearance with a whitish haze. A paint sample was taken from the photo-degraded yellow paint in the citron and its layer structure and composition were investigated with light microscopy and backscatter electron imaging combined with elemental analyses (Fig. 5a-l). The sample comprises a colored ground layer composed of chalk and red earth pigment (Fig. 5b, layer 1). This is followed by a dark brown underlayer with a mixture of large lead white particles, fine black, red earth pigment and chalk (layer 2). Three paint layers were applied, an orange layer (layer 3) mainly pigmented with earth pigments, a thin transparent light yellow layer (layer 4) containing lead white, orpiment, lead tin yellow and gypsum, and a thick yellow layer (layer 5) composed of orpiment, gypsum, 

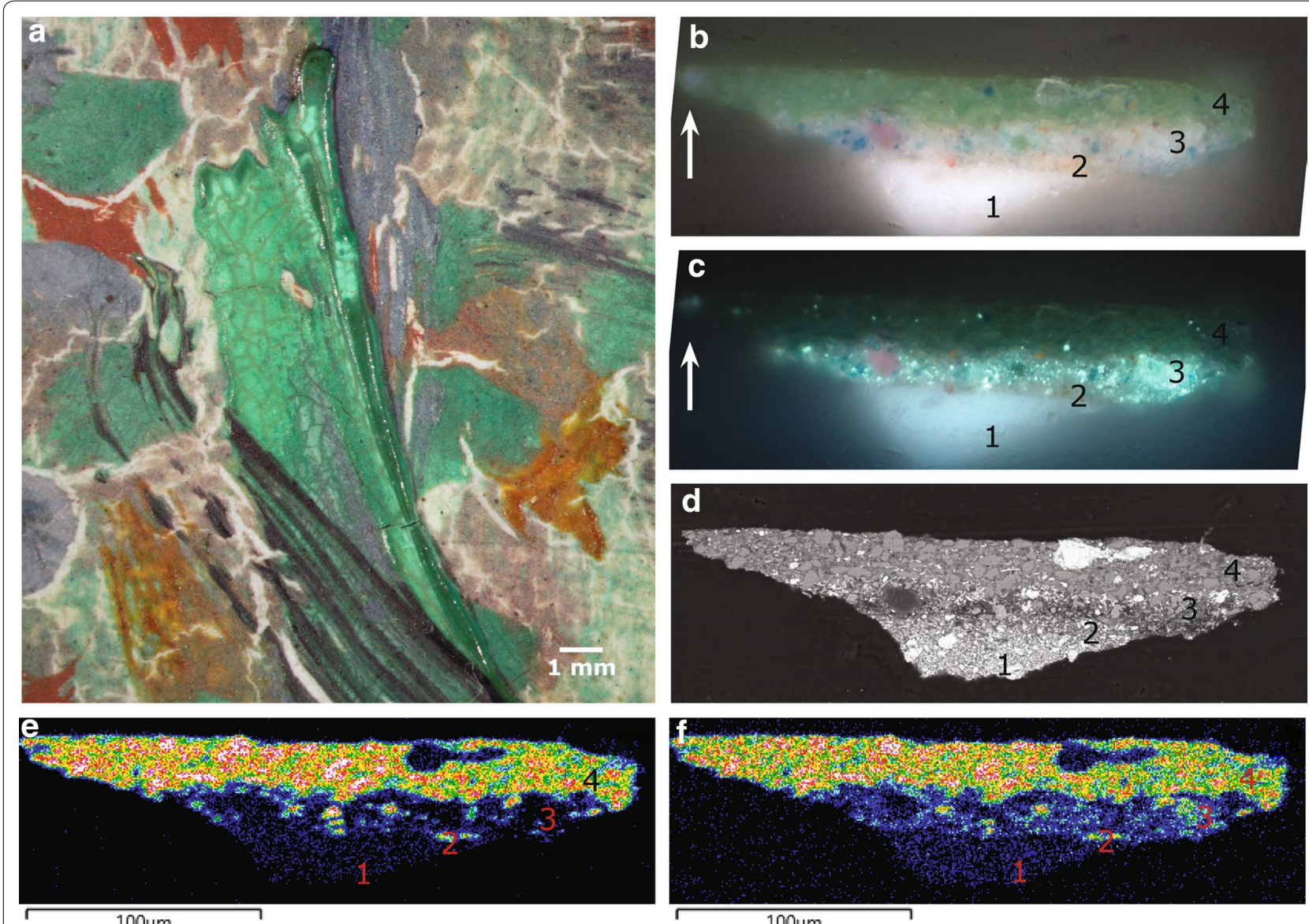

$100 \mu \mathrm{m}$
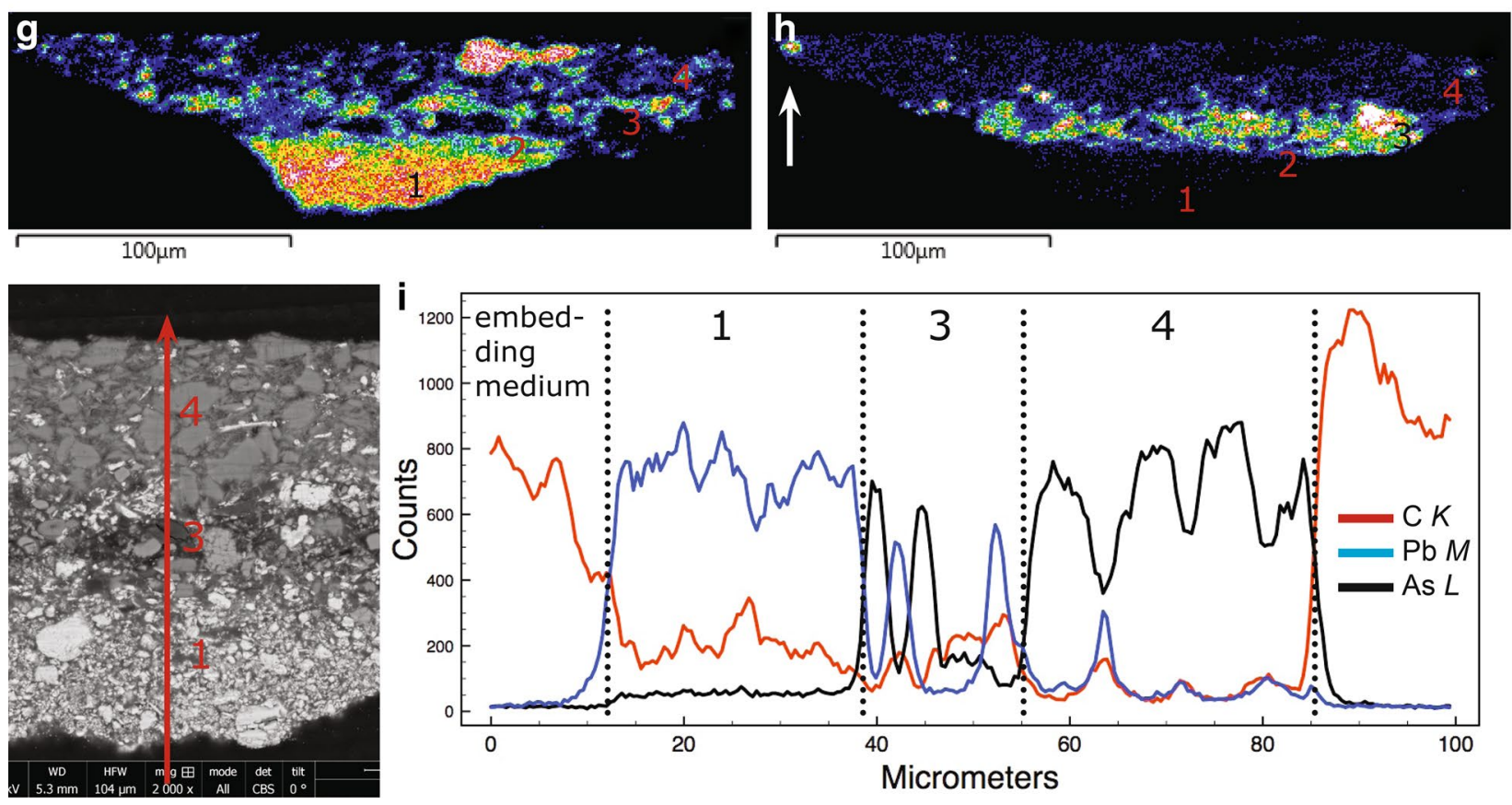
(See figure on previous page.)

Fig. 3 Detail of the Interior of a Restaurant (1887) by Vincent van Gogh showing the lightened green paint (a) (photograph: Kröller-Müller Museum), light microscopic image of paint sample taken from a lightened green area, normal light (b) and UV (c); backscatter electron image (d), elemental maps of arsenic $(\mathbf{e})$, copper $(\mathbf{f})$, lead $(\mathbf{g})$, zinc (h) and line scan revealing the carbon (red line), lead (blue line) and arsenic (black line) X-ray intensities (i). The paint sample consists of a lead white-containing ground (layer 1), a thin beige layer containing lead white and fine red and oranges pigments (layer 2), a purple layer with lead white, zinc white, red lake with Al-substrate, cerulean blue, emerald green, barium sulfate and fine yellow, orange and red pigment (layer 3), a green paint layer (layer 4) composed of emerald green, lead white, a little zinc white and a few yellow, orange and blue particles. Note: the paint sample was not taken from the lightened green paint shown in $\mathbf{a}$, but from a comparable brush stroke in the painting

silicates and a little lead white. The silicates are possibly finely ground glass added as a drier. The lower part of layer 5 is still bright yellow, while the upper part looks more transparent. The orpiment particles are missing in the upper part of this layer, indicating photo-degradation (Fig. 5a). A white highlight (layer 6) of lead white layer is partly present in this sample. Finally, there is a varnish layer (layer 7). Elemental analyses detected arsenic in all paint layers, with relatively higher concentrations in layers 4, 5 and 6 (Fig. 5j). Interestingly, at the upper part of layer 2 and in layer 6-both layers containing lead white- $\mathrm{As}-\mathrm{Pb}$ rich needles are visible in the backscatter electron image (Fig. 5d). Lead arsenates are not expected to have been present originally in the paint sample. These needles indicate the presence of newly formed reaction products inside the paint layer.

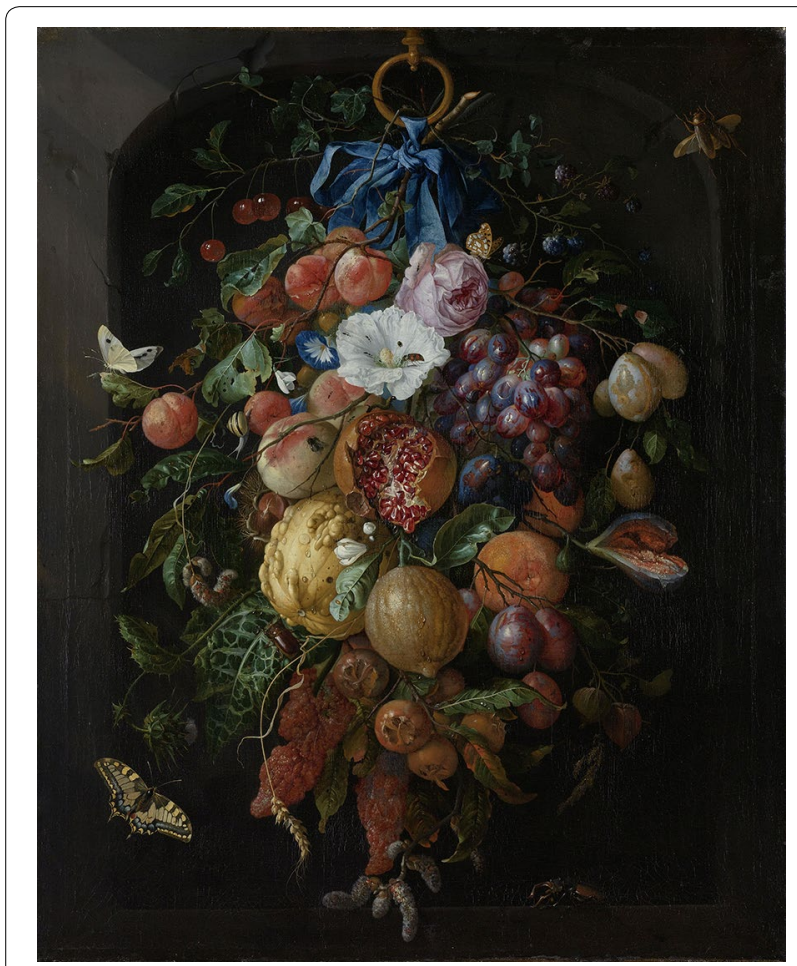

Fig. 4 Festoon of Fruits and Flowers (1660-1670), Jan Davidsz. de Heem, Rijksmuseum Amsterdam (SK-A-138), Netherlands
A sum of many ATR-FTIR spectra taken from the layer 6 (green line) and layer 3 (blue line) of the de Heem sample reveal a strong broad band at $791 \mathrm{~cm}^{-1}$ indicative of an arsenate vibration (Fig. 5q) (for reference spectra see ref. [5]). A weak band at $790 \mathrm{~cm}^{-1}$ was observed in the sum of many spectra taken from layers 1 and 5 . Imaging ATR-FTIR maps show the distribution of a C-O band at $1722 \mathrm{~cm}^{-1}$ representative for the embedding medium (Fig. $5 \mathrm{~m}$ ), an As-O band at $791 \mathrm{~cm}^{-1}$ representative for arsenates (n), a $\mathrm{Si}-\mathrm{O}$ band at $1072 \mathrm{~cm}^{-1}$ representative for historical glass (o) and a $\mathrm{Ca}-\mathrm{O}$ band at $1400 \mathrm{~cm}^{-1}$ representative for carbonates (p). The map at $1722 \mathrm{~cm}^{-1}$ outlines the paint sample. The carbonates are dominant in the ground layer and are present in relatively lower intensities in layers 2 and 5 . The distribution of the carbonates matches the calcium map (f). The silicate distribution ( $\mathrm{Si}-\mathrm{O}$ band, o) corresponds to the silicon map (h) and is found in the earth-pigmented layer 3 and in the orpiment layer 5 . The arsenates are found at relatively low intensity throughout the paint sample and with relatively high intensities at the interface between layers 2 and 3 and layers 4 and $6(\mathrm{n})$. The fact that the arsenate is found at the interface between layers 2 and 3 and not in relatively high concentrations throughout layer 2 indicates that the arsenic is 'trapped' in the upper part of paint layer 2. A similar observation was made in a degraded orpiment/realgar paint sample taken from Still Life with Five Apricots by Adriaen Coorte discussed by Keune et al. 2015 [5]. In general, as arsenates are also predominantly present in layer 6 , we can conclude that arsenic is also accumulated in lead rich areas.

K-edge XANES analyses carried out on the de Heem sample identified species containing As(III) and As(V). Figure $5 \mathrm{r}$ shows the XANES data taken from an intact arsenic sulfide particle in the lower part of the paint layer (layer 5, red line), the upper part of the degraded paint layer (layer 5 , blue line), the calcium-containing ground layer (layer 1 , green line) and the top layer containing lead white (layer 6, black line). As expected the most reduced arsenic species in the paint sample, arsenic sulfide, is found in the intact particle (white line at $11,870.0 \mathrm{eV}$, red line). The edge position matches the XANES of realgar and orpiment references (XANES references, see ref. [4]) reasonably well. The most oxidized arsenic species 

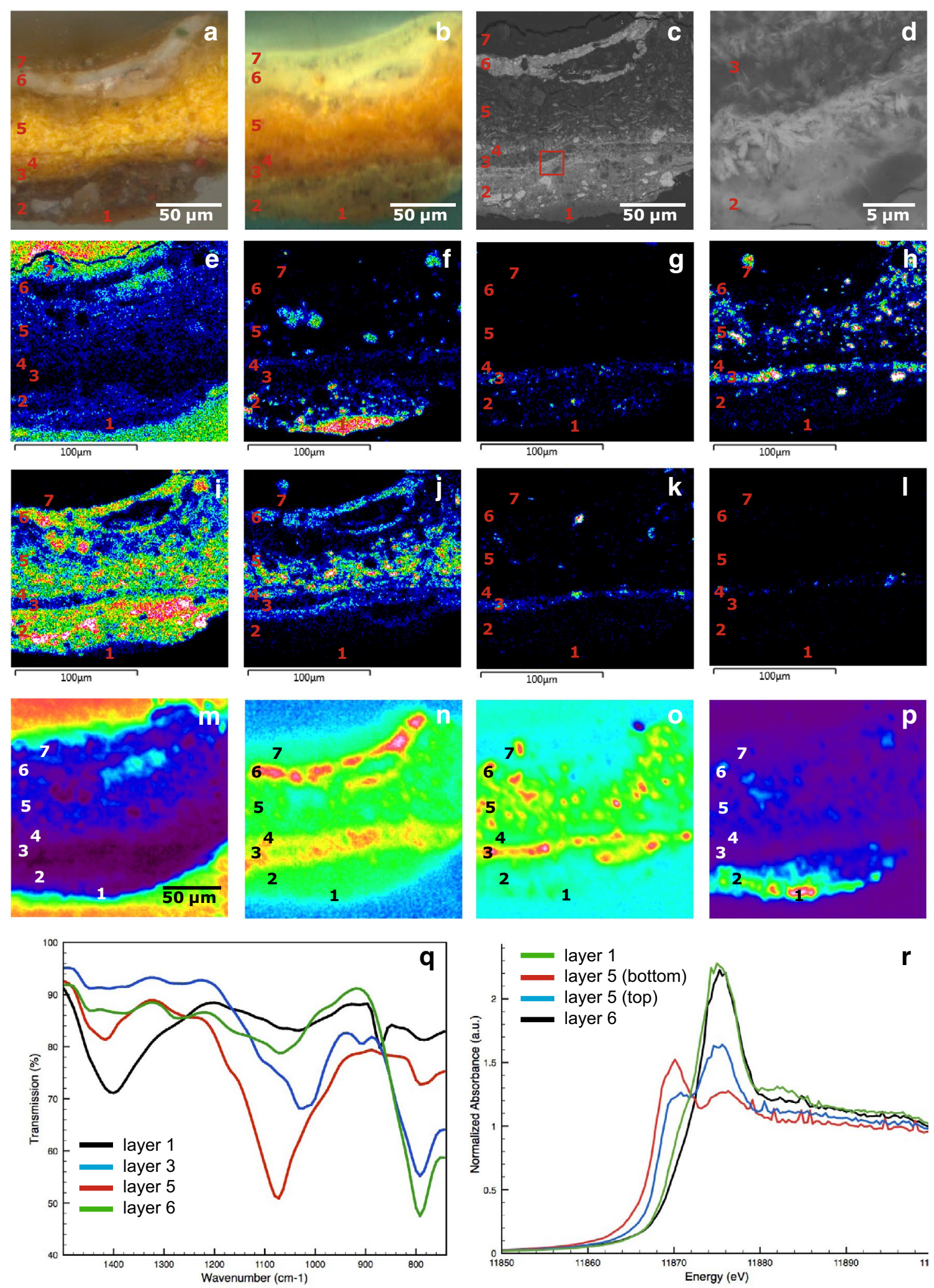
(See figure on previous page.)

Fig. 5 Detail of light microscopic image of the Heem sample under white light (a), ultraviolet (b), backscatter electron image (c) and detail d showing layers 3-4, the detail is indicated with a red box in $\mathbf{c}$. Elemental maps of carbon (e), calcium $(\mathbf{f})$, iron $(\mathbf{g})$, silicon $(\mathbf{h})$, lead (i), arsenic $(\mathbf{j})$, aluminum $(\mathbf{k})$, and tin (I) are obtained with the SEM/EDX. False color-plots ATR-FTIR images at $1722 \mathrm{~cm}^{-1}(\mathbf{m}), 791 \mathrm{~cm}^{-1}(\mathbf{n}), 1028 \mathrm{~cm}^{-1}(\mathbf{o})$, and $1400 \mathrm{~cm}^{-1}(\mathbf{p})$ (red is representative for high intensities). Part of ATR-FTIR spectrum of a sum of many spectra from the ground (layer 1, black line), iron-containing layer (layer 3, blue line), orpiment layer (layer 5, red line) and lead white layer (layer 6, green line) (q). Arsenic -K-edge XANES spot analyses taken from an intact arsenic sulfide particle in the lower part of the paint layer (layer 5, red line), upper part of the degraded paint layer (layer 5, blue line), calcium-containing ground layer (layer 1, green line) and the lead white-containing top layer (layer 6, black line) (r)

is found in the ground and the top layer (layers 1 and 6) (white line at $11,875.5 \mathrm{eV}$ ). These spectra match the spectra of the arsenate $\left(\mathrm{As}^{5+}\right)$ species. The reduced and oxidized species are present in the upper part of the orpiment-containing layer (layer 5). The reduced arsenic derives from the intact arsenic sulfide pigment or arsenite $\left(\mathrm{As}^{3+}\right)$, which is the photo-degradation product of orpiment.

In order to obtain more details about the various arsenic species, a linear combination least squares (LCLS) fit of the four XANES was carried out using reference XANES for realgar, $\mathrm{As}_{2} \mathrm{O}_{3}$, and $\mathrm{As}_{2} \mathrm{O}_{5}$ (Fig. 6). Here it should be noted that in this fit the references were not forced to sum to 1 (100\%) accounting for the fact that the fitting model might not include all species present in the sample. However, the fit produced reasonably linear combinations summing to $93.6,88.6,92.2$, and $100 \%$, and R-factors of $0.0106,0.0167,0.0108$, and 0.0062 for layers 6, 5 (bottom), 5 (top), and 1 respectively. As expected, the XANES taken from the bottom of layer 5 is dominated by realgar, and realgar is lacking in layers 1 and 6 . Interestingly, $\mathrm{As}^{3+}$ dominates over $\mathrm{As}^{5+}$ in layer 5 , while $\mathrm{As}^{5+}$ is the main species in layers 1 and 6 . This indicates that further away from the original arsenic-containing pigment,

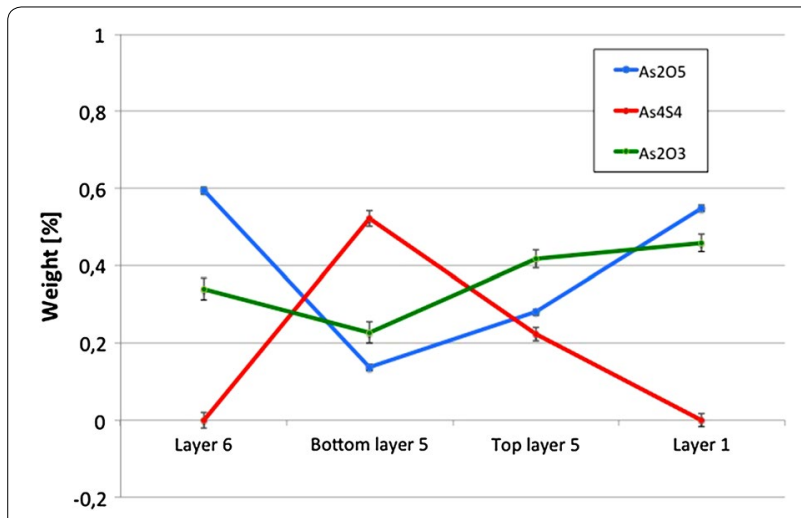

Fig. 6 Fitting of the arsenic -K-edge XANES spot analyses taken from an intact arsenic sulfide particle in the lower part of the paint layer (bottom layer 5), upper part of the degraded paint layer (top layer 5), calcium-containing ground layer (layer 1 ) and the lead white-containing top layer (layer 6) fitted with realgar, $\mathrm{As}_{2} \mathrm{O}_{3}$ and $\mathrm{As}_{2} \mathrm{O}_{5}$ references
$\mathrm{As}^{5+}$ is the most stable arsenic species, while the $\mathrm{As}^{3+}$ species is still present in the paint.

\section{Case study: The Descent of the Cattle, nineteenth century- emerald green}

Most emerald green pigments in The Descent of the Cattle (1836) by Théodore Rousseau in the Mesdag Collection in The Hague have been degraded (Fig. 7). The layer structure, composition and elemental distribution of The Descent of the Cattle paint sample can be found in Table 1 and ref. [1] (sample 286-8A). In this paper, the SR- $\mu$ XRFbased elemental maps of $\mathrm{As}, \mathrm{Cu}, \mathrm{Fe}, \mathrm{Cr}$ and $\mathrm{Ca}$ taken from a detail of The Descent of the Cattle are presented (Fig. 8a-f). This area encloses layers 3-16 as described. Arsenic is dispersed over the entire sample, with hotspots in layers 6, 8-10, and 14 (Fig. 8b). These hotspots

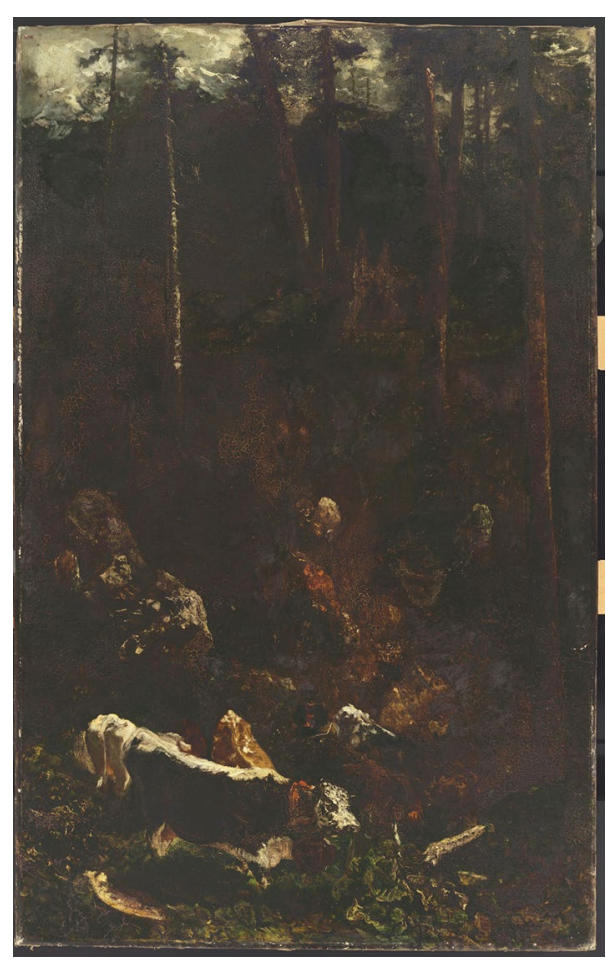

Fig. 7 The Descent of the Cattle by Théodore Rousseau (oil on canvas, $259 \times 162 \mathrm{~cm}$ ) (photograph: Van Gogh Museum, Amsterdam, 2011) 
Table 1 Layer structure and composition of The Descent of the Cattle paint cross-section

\begin{tabular}{llll}
\hline Layer & Color appearance & Thickness (microns) & Identified components \\
\hline 16 & Yellowish brown & $10-50$ & Varnish constituents \\
15 & Yellowish brown & 20 & Varnish constituents \\
14 & Orangish & $20-30$ & Vermilion, red lake (Al), silicates, gypsum, starch, iron oxide \\
13 & Greenish & $10-20$ & Prussian blue, bone black, lake \\
12 & $10-15$ & Vermilion \\
11 & Blue-black & $10-20$ & Carbon particles, Prussian blue, silicate particles, chrome yellow, \\
& Greenish & & Kassel earth, barium sulfate \\
9 & Greenish & $20-30$ & Copper carboxylates \\
8 & Green & $10-30$ & Copper carboxylates \\
7 & Yellow & 10 & Yellow ochre, Prussian blue, emerald green \\
6 & Translucent green brown & 10 & Yellow ochre, vermilion, carbon brown \\
5 & Dark brown & $30-40$ & Emerald green, yellow lake (Al), chrome yellow, barium sulfate \\
4 & Dark brown & $20-50$ & and lead white \\
3 & Reddish brown & $10-40$ & Unknown \\
2 & Dark brown & $>80$ & Organic rich, lead drier \\
1 & White & $20-25$ & Vermilion, ochre, lead white, (black or brown) carbon, lake (Al), \\
& & $>25$ & green earth \\
\hline
\end{tabular}

correlate with the hotspots visible in the copper map, and must be As- $\mathrm{Cu}$ rich particles. The iron is present in relative high intensities in layers 7 and 8 , i.e. yellow and green earth pigmented layers. Iron and chromium are found in layer 11, while chromium is also detected in the layer containing Emerald green, layer 6. Calcium dominates in layers 13 and 14, layers that contain lakes (with a calcium substrate) and gypsum.

Arsenic K-edge XANES analyses carried out on The Descent of the Cattle sample identified species containing both $\mathrm{As}^{3+}$ and $\mathrm{As}^{5+}$. Figure 8i shows the XANES taken from the emerald green pigment (reference sample, black), an As-Cu rich particle in the paint sample (layer 6, magenta), the iron-containing ground layer (layer 3, green), the copper rich layer (layers 9-10, red), and the varnish (layer 15-16 blue). The white line of the emerald green pigment is found at $11,871.8 \mathrm{eV}$, but the $\mathrm{As}-\mathrm{Cu}$ rich particle has peaks at both $11,871.9$ and $11,875.2 \mathrm{eV}$. The first peak in the XANES of the As-Cu particle matches the white line of the emerald green reference and is interpreted as an intact emerald green pigment particle. As the XANES represents the bulk of the paint sample (no thin section was made), we expect that the white line at $11,875.2 \mathrm{eV}$ originates from the degradation product of the emerald green surrounding the particle. The ironcontaining ground as well as the copper-containing layers both have white lines at $11,875.4 \mathrm{eV}$, while the white line of the varnish lies at $11,875.7 \mathrm{eV}$. The main arsenic species in The Descent of the Cattle sample is $\mathrm{As}^{5+}$, an arsenate. The slight difference in the position of the white line is thought to be caused by different counter-ions. Lead is the dominating cation present in the varnish and can act as a counter ion forming lead arsenates [1]. Iron arsenates are most likely to be formed in the iron layer, but lead arsenates cannot be excluded. Besides copper, the copper-containing layer also has lead, calcium, and potassium, so presumably lead and/or calcium arsenates are present. As in previous research, arsenic trioxide has been proposed as the main degradation product, and these results show that arsenates are the final end product of the chemical degradation of emerald green.

To gain a better understanding of the diffuse nature of the arsenic degradation products, multiple $\mu$-XRF maps were collected while tuning the X-ray incidence energy across the As K-edge. This generates XANES data for each pixel in the map obtained, while using a reduced number of energy points results in significant time saving during measurement. These multi-energy maps clearly indicate the presence of multiple arsenic species. In total 30 maps were collected, with varying energy step sizes between 5 and $0.5 \mathrm{eV}$ from 11,845 to $11,960 \mathrm{eV}$ (smaller energy steps were used across the white line). We performed a standard XANES image analysis with this limited XANES data set using the XANES-Wizard software, which is part of the TXM-Wizard software package [24-26]. In the first step the edge energy, which is indicative of the oxidation state of arsenic, has been determined for each single pixel in the map by identifying the energy at which the normalized XANES intensity was equal to half the edge jump. The result is displayed in Fig. $8 \mathrm{~g}$, where red colors 

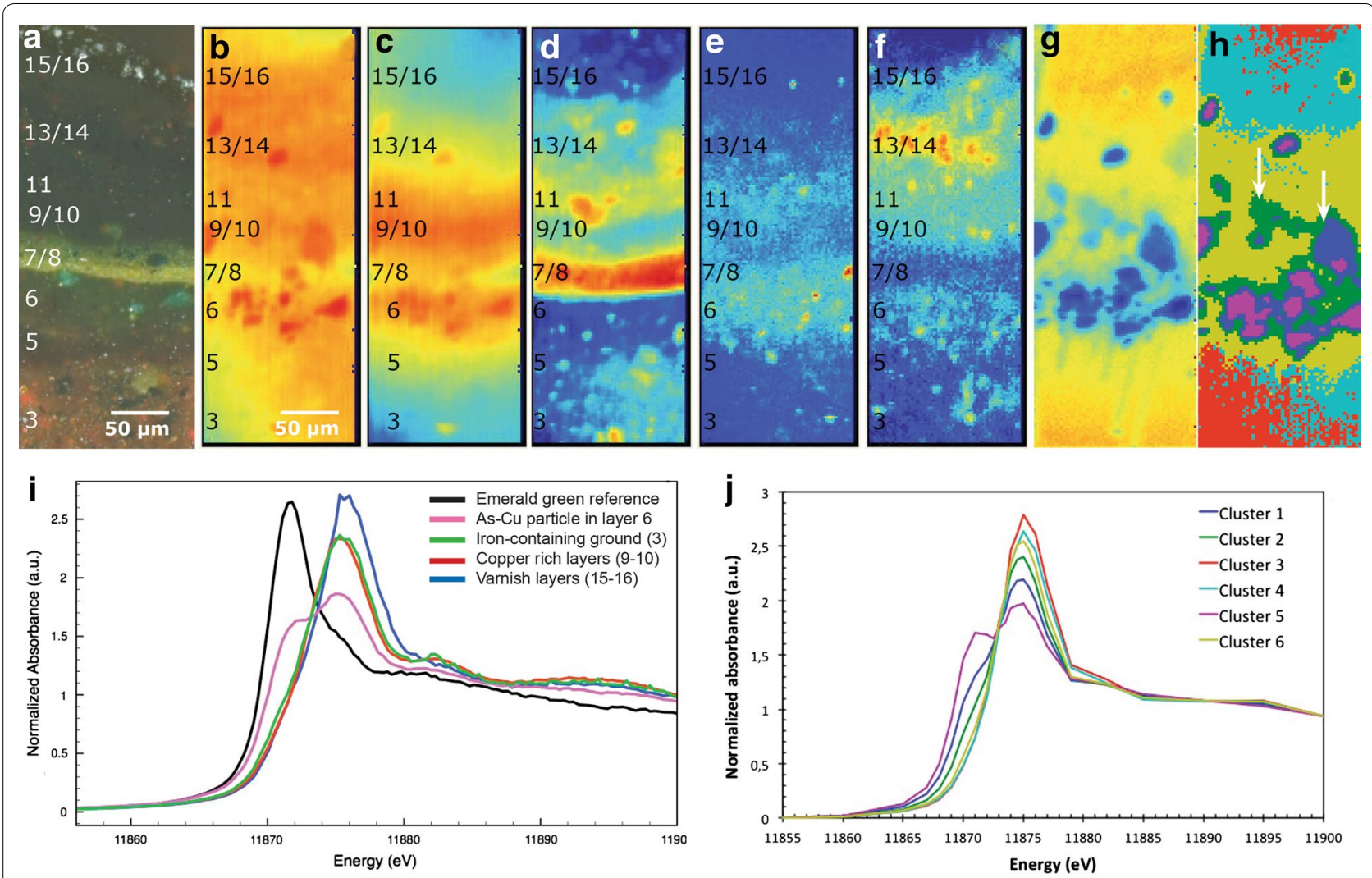

Fig. 8 Detail of light microscopic image of The Descent of the Cattle sample (sample code: 286-A) under white light (a), the detail corresponds to the area measured for the $\mu$-XRF and multi-energy maps. Elemental distribution maps of arsenic (b), copper (c), iron (d), chromium (e), and calcium (f) obtained with $\mu$-XRF (data collection at SSRL BL 2-3; $\mu$-XRF maps excited at 11,960 eV, map size $(\mathrm{v} \times \mathrm{h}$ ): $342 \times 128 \mu \mathrm{m}$ ). The layer composition and layer structure of the paint sample can be found in Table 1 and in Keune et al. [1]. The distribution of the As K-edge position where blue pixels indicate more reduced and orange/red pixels more oxidized As $(\mathbf{g})$ and image segmentation based on PCA and k-means clustering (h). Arsenic -K-edge XANES spot analyses taken from emerald green reference sample (black), As-Cu rich particle (layer 6, magenta), iron-containing ground layer (layer 3, green), copper rich layer (layer 9-10, red) and varnish layer (layer 15-16, blue) (i). Average XANES of clusters 1-6 corresponding to the map shown in $\mathbf{h}$ (j). Note that the light microscopic image visualizes the surface and $\mu$-XRF and multi-energy maps the paint volume of the sample

indicate more oxidized species, while blue represents more reduced species. The most reduced areas match with the arsenic-rich and copper-rich hotspots, and the oxidized arsenic covers the rest of the paint sample. The next step was a Principal Component Analysis (PCA) and subsequent k-means clustering of the XRF-XANES data sets (as described for example in refs. [24, 25]). The first three principal components (PC) covered $90.75 \%$ of the variance in the data and were therefore used for image segmentation by k-means clustering. K-means clustering was performed in this 3-dimensional PC space using seven clusters (Fig. 8h), intentionally over-clustering the data set to obtain an image segmentation into 7 regions of XANES similarity. The average XANES spectra for the clusters are reported in Fig. 8j. Cluster 7 captured the very few pixels that had low signal to noise ratios and their average XANES have therefore not been used in the following discussion. Cluster 5 (purple) represents the most reduced arsenic species in the paint sample (peak at $11,871 \mathrm{eV})$. The spatial distribution of this cluster fits with $\mathrm{As}-\mathrm{Cu}$ (emerald green) particles. This reduced arsenic species is diminished in cluster 1 (blue), and is hardly visible in cluster 2 (green). On the contrary, the most oxidized arsenic species (white line at 11,875 eV) increases in these clusters and increasingly dominates clusters 2 (green), 6 (yellow), 4 (cyan), and 3 (red). This clustering is exclusively based on spectral similarity. It is therefore interesting to note that this trend is also visible in the spatial distribution of these clusters. The further away from the emerald green particles, the more oxidized species were found. So $\mathrm{As}^{3+}$ can be still found close to the pigment, but the $\mathrm{As}^{3+}$ oxidizes close to its original sources and $\mathrm{As}^{5+}$ is found throughout the system. This points to the migration of the arsenic as an $\mathrm{As}^{5+}$ species. We never see pure $\mathrm{As}^{3+}$ because we measure the paint volume and always find some contribution of $\mathrm{As}^{5+}$ surrounding the particle. The spatial distribution of $\mathrm{As}^{3+/ 5+}$ also tells us about the presence of partially degraded emerald green 
pigments. In the hotspots indicated with a white arrow in Fig. 8h, cluster 5 (most reduced) is absent and clusters 1 (blue) and 2 (green) are mainly present, i.e. clusters with a relatively low $\mathrm{As}^{3+}$ species contribution.

\section{Conclusions}

All paint samples presented illustrate that arsenic migrates throughout the whole depth of the multi-layered paint structure of the artwork, from the wood substrate to top varnish layers. The De Heem sample indicates that arsenic has a high affinity for lead-containing layers resulting in lead-arsenic rich crystalline needles, interpreted as lead arsenates. Lead arsenates are typically seen as opacifiers in glasses, and this is the first time that they are observed to form as paint degradation products. The emerald green particles might seem visually to be intact in the Van Gogh and the Austrian cupboard samples. Nevertheless, relatively low yields of arsenic are found throughout the paint sample indicating minor degradation of emerald green occurring in the area surrounding these particles. The photo-degradation of orpiment and realgar and the chemical degradation of emerald green have the arsenic-containing degradation products in common. XANES of the De Heem and The Descent of the Cattle samples revealed $\mathrm{As}^{3+}$ in and close to the intact particles, while at larger distances $\mathrm{As}^{5+}$ is present. It was not possible to distinguish between arsenic trioxide and emerald green with XANES because in both cases arsenic has a $3+$ valence. It is clear that the most favorable and stable form of the arsenic degradation product in paint is arsenate. Further research is required to understand the extent of any lateral migration of the arsenic.

It is important for the conservator to be aware that in objects where paints contain orpiment, realgar and emerald green, arsenic is deposited throughout the entire object, including wood support and top varnish layers, forming new arsenic-containing products inside the paint. This has implications for the safe handling of works of art during conservation and also the preservation of original material if varnishes are to be removed. The migration of arsenic is a water-based transport mechanism [5] so the use of water-based cleaning agents and large fluctuations in relative humidity $(\mathrm{RH})$ should be minimized in order to reduce arsenic migration.

\section{Experimental \\ Embedding}

The samples were embedded in a polyester resin (Polypol) and dry polished with Micro-mesh ${ }^{\circledR}$ polishing cloths (final step 12000 mesh).

\section{Light microscopy}

All paint cross-sections were examined under a Zeiss Axioplan 2 microscope, with both incident polarized light and incident UV light (from a xenon-lamp and a mercury short arc photo optic lamp $\mathrm{HBO}$, respectively). The UV H365 filter set used for examination in UV light consists of the following filters: excitation BP 365/12, beam splitter FT 395 and emission LP 397.

\section{Sem/edx}

Scanning electron microscopy in combination with energy dispersive X-ray analysis (SEM-EDX) studies were performed on a Verion high vacuum electron microscope (FEI, Eindhoven, Netherlands) with an EDX system with spot analysis and elemental mapping facilities (Oxford). Backscattered electron images of the cross-sections were taken at a $20 \mathrm{kV}$ accelerating voltage, at a $5 \mathrm{~mm}$ eucentric working distance and with current density of approximately $130 \mathrm{pA}$. Samples were gold coated (3 nm thickness) in an SC7640 gold sputter coater (Quorum Technologies, Newhaven, East Sussex, UK) prior to SEMEDX analysis to improve surface conductivity.

\section{Imaging ATR-FTIR}

The FTIR spectral data were collected on a Perkin Elmer Spectrum 100 FTIR spectrometer combined with a Spectrum Spotlight 400 FTIR microscope equipped with a $16 \times 1$ pixel linear mercury cadmium telluride (MCT) array detector. A Perkin Elmer ATR imaging accessory consisting of a germanium crystal was used for ATR imaging.

\section{As-K-XANES}

BL2-3 at SSRL is a bending magnet side station dedicated to X-ray imaging and micro X-ray absorption spectroscopy operating at an energy range from 5 to $24 \mathrm{keV}$. A Kirkpatrick-Baez mirrors system is used to achieve micro-focus with a beam size of $\sim 2 \times 2 \mu \mathrm{m}^{2}$. The microscope consists of a double crystal monochromator [Si (111)] used for energy selection and scanning of the X-ray energy. Beamline $2-3$ is equipped with a Vortex silicon drift detector and ionization chambers. Sample positions are controlled with submicron accuracy by a Newport Micro sample positioner. The samples were placed with double sided adhesive tape (Scotch ${ }^{\circledR}$ tape) in the sample holder and measured under ambient conditions and room temperature. Prior to the analysis of the paint cross-sections, powdered reference samples (described below) were prepared for bulk XANES analysis by dusting the finely ground powder onto sulfur-free Kapton tape. Cross-section samples were kept in a vertical plane at $90^{\circ}$ to the incident beam.

\section{Principal component analysis (PCA) and k-means clustering}

PCA was carried out using the mean-centered data matrix (consisting of $\mathrm{p}$ pixels and E energies) and singular 
value decomposition (SVD) reducing the dimensionality of the data set from $\mathrm{E}$ to $\mathrm{N}$ dimensions. This reduction is achieved by using only the first $\mathrm{N}$ principal components (PCs), which explain most of the data's variance, but without losing significant information because higher PCs describe mainly the noise in the data set. Therefore, and also because the PCs are oriented to best describe the spread in the data, projecting the data to this $\mathrm{N}$-dimensional principal component space highlights the pattern explained by the captured variance and effectively reduces noise. In this reduced space the distance between data points is a direct measure of the similarity of the XANES, i.e. the chemical phase, and can be used to cluster pixels according to their (euclidean) distances from cluster centers (centroid linkage method, k-means clustering). This results in an effective grouping of pixels with similar XANES into k groups resulting in image segmentations. The number of clusters $k$ has to be at least equal to $\mathrm{N}$ (in this study 3 ) in order to exploit all the information provided by the reduced PC space, but the data was intentionally over-clustered using $\mathrm{k}=7$ to achieve a finer image segmentation in order to inspect various different stages of oxidation state transitions. A more detailed description of the applied approach to process XANES image data can be found in references [24, 25].

\section{Authors' contributions}

KK: analytical strategy, selection of the samples, data acquisition, analysis and interpretation of all samples, data processing, drafting manuscript; JM: conservation context of the degradation of arsenic pigment in paintings and painted objects, drafting manuscript; AM: synchrotron radiation X-ray microscopy beamline support; $\mathrm{JH}$ : synchrotron radiation $\mathrm{X}$-ray microscopy analyses; $\mathrm{FM}$ : data processing, synchrotron radiation X-ray microscopy beamline support; All authors read and approved the final manuscript.

\section{Author details}

${ }^{1}$ Rijksmuseum Amsterdam, Conservation and Restoration, PO BOX 74888, 1070, DN, Amsterdam, The Netherlands. ${ }^{2}$ Van 't Hoff Institute for Molecular Sciences, University of Amsterdam, Science Park 904, 1098, XH, Amsterdam, The Netherlands. ${ }^{3}$ Conservation Department, Scientific Research and Analysis Laboratory, Winterthur Museum, Winterthur, DE, USA. ${ }^{4}$ Stanford Synchrotron Radiation Lightsource, SLAC National Accelerator Laboratory, 2575 Sand Hill Rd, Menlo Park, CA, USA. ${ }^{5}$ Department of Materials Science and Engineering, University of Delaware, Newark, DE, USA. ${ }^{6}$ Inorganic Chemistry and Catalysis, Debye Institute for Nanomaterials Science, Utrecht University, Universiteitsweg 99, 3584, CG, Utrecht, The Netherlands.

\section{Acknowledgements}

This work is part of the PAinT project, supported by the Science4Arts program of the Dutch Organization for Scientific Research (NWO). Some of the measurements reported here were performed on beamline 2-3 at the Stanford Synchrotron Radiation Lightsource, a Directorate of SLAC National Accelerator Laboratory and an Office of Science User Facility operated for the U.S. Department of Energy's Office of Science by Stanford University. The authors are very grateful to René Boitelle (Van Gogh Museum, Netherlands), Lara van Wassenaer (private conservator, Amsterdam), Arie Wallert (Rijksmuseum Amsterdam), Matthijs de Keijzer (Cultural Heritage Agency of the Netherlands) and Margje van Leeuwestein (Kröller-Müller Museum, Otterlo) for providing the paint samples. They would like to thank Sam Webb (SSRL, USA), Susan Smelt (Rijksmusuem Amsterdam) and Sydney Beall (Rijksmuseum Amsterdam) for their technical support.

\section{Competing interests}

The authors declare that they have no competing interests.

Received: 18 September 2015 Accepted: 30 March 2016

Published online: 21 April 2016

\section{References}

1. Keune K, Boon JJ. Degradation of emerald green in oil paint and its contribution to the rapid change in colour of the Descente des vaches (1834-35) painted by Théodore Rousseau. Stud Conserv. 2013:58:199-210.

2. Hoshi E, Kitada M. Microstructures and decomposition of Cu-containing green pigment used in the late edo era. J Japan Inst Metals. 2009;73:387-93.

3. Pottasch C, Mensch K. Arsenic and apricots: understanding the sober still lifes of Adriaenn Coorte. In: Meeks N, Cartwright C, Meek A, Mongiatti A, editors. Historical technology, materials and conservation: SEM and microanalysis. Archetype Publications: London; 2012. p. 100-6.

4. Mahon D, Centeno SA, Wypyski MT, Salomon XF, Bayer A. Technical study of three allegorical paintings by Paolo Veronese: the choice between virtue and vice, wisdom and strength, and mars and venus united by love. In: Metropolitan museum studies in art, science, and technology; 2010. p 83-108.

5. Keune K, Mass J, Meirer F, Pottasch C, van Loon A, Hull A, Church J, Pouyet E, Cotte M, Mehta A. Tracking the transformation and transport of arsenic sulfide pigments in paints: synchrotron-based X-ray micro-analysis. J Anal At Spectrom. 2015;30:813-27.

6. Douglass DL, Shing C, Wang G. The light-induced alteration of realgar to pararealgar. American Mineralolist. 1992;77:1266-74.

7. Ballirano P, Maras A. Preliminary results on the ligh-induced alteration of realgar: kinetics of the process. Plinius. 2002;28:35-6.

8. Mass J. Personal observations; 2015.

9. Trentelman K, Stodulski L, Pavlosky M. Characterization of pararealgar and other light-induced transformation products from realgar by Raman microspectroscopy. Anal Chem. 1996;68:1755-61.

10. Rötter C, Grundmann G, Richter M, van Loon A, Keune K, Boersma A, Rapp K. The occurrence of artificial orpiment (dry process) in northern European painting and polychromy and evidence in historical sources. In: Schuller M, Emmerling E, Nerdinger W, Verlag Anton Siegl, editors. Auripigment/Orpiment: Studien zu dem Mineral und den künstlichten Produkten. Fachbuchhandlung $\mathrm{GmbH}$. München; 2007.

11. van Loon A: Colour changes and chemical reactivity in seventeenth-century oil paintings. In: Ph.D. Thesis. University of Amsterdam, Molart Series (14), AMOLF: Amsterdam; 2008.

12. Sheldon L, Woodcock S, Wallert A. Orpiment overlooked-expect the unexpected in 17th century workshop practice. In: Verger I, James \& James, EDITORS. ICOM Committee for Conservation. vol 1. 14th Triennial Meeting: The Hague; 2005. p 529.

13. Vermeulen M, Sanyova J, Janssens K. Identification of artificial orpiment in the interior decorations of the Japanese tower in Laeken, Brussels, Belgium. Herit Sci. 2015;3:9.

14. Rötter C. Auripigment. Restauro. 2003;6:408-13.

15. Dubois H, Meloni S, Metz E, Schoonhoven B, Wallert A. Processes of orpiment and realgar-based sixteenth-century paints. In: Phenix A, UKIC, editors. Deterioration of artists' paints: effects and analysis - a joint meeting of ICOM-CC working groups paintings 1 and 2 and the painting section. London: British Museum; 2001. p. 75-97.

16. Hendriks $E$, Wallert A. Orpiment used in paintings by Maarten van Heemskerck (1498-1574): degradation of the pigment and related conservation problems. In: Goupy J, Mohen JP, CNRS editors. Art et Chimie, la Couleur: Actes du Congrès. Paris; 1998. p 111-2.

17. Fitzhugh EW. Orpiment and realgar. In: Fitzhugh EW, editor. Artists' pigments, a handbook of their history and characteristics, vol. 3. Oxford: Oxford University Press; 1997. p. 47-80.

18. Wallert A. Orpiment and realgar. Maltechnik/Restauro. 1984;90:45-57.

19. Welch AH, Stollenwerk KG, editors. Arsenic in ground water; geochemistry and occurrence. Boston: Kluwer Academic Publishers; 2003. 
20. Castro K, Sarmiento A, Princi E, Perez-Alonso M, Rodriguez-Laso MD, Vicini S, Madariaga JM, Pedemonte E. Vibrational spectroscopy at the service of industrial archaeology: nineteenth-century wallpaper. Trends Anal Chem. 2007;26:347-59.

21. Brown WL. Arsenic and wallpaper. Br Med J. 1932;1:772.

22. Jones DEH, Ledingham KWD. Arsenic in Napoleon's wallpaper. Nature. 1982;299:626-7.

23. Fiedler I, Bayard MA. Emerald green and Scheele's green. In: Fitzhugh EW, editor. Artists' pigments, a handbook of their history and characteristics, vol. 3. Oxford: Oxford University Press; 1997. p. 219-71.

24. Liu Y, Meirer F, Williams P, Wang J, Andrews JC, Pianetta P. TXM-Wizard: a program for advanced data collection and evaluation in full-field transmission X-ray microscopy. J Synchrotron Radiat. 2012;19:281-7.
25. Meirer F, Liu Y, Pouyet E, Fayard B, Cotte M, Sanchez C, Andrews JC, Mehta A, Sciau P. Full-field XANES analysis of Roman ceramics to estimate firing conditions - a novel probe to study hierarchical heterogeneous materials. J Anal At Spectrom. 2013;28:1870-83.

26. Boesenberg U, Meirer F, Liu Y, Khushalchand Shukla A, Dell'Anna R, Tyliszczak T, Chen G, Andrews JC, Richardson TJ, Kostecki RM, Cabana J. Mesoscale phase distribution in single particles of $\mathrm{LiFePO}_{4}$ following lithium deintercalation. Chem Mater. 2013;25:1664-72.

27. Keune K, Boon JJ, Can dispersed and migrated arsenic from degraded pigments in paintings be a marker for water-linked transport processes?. In: ICOM Committee for Conservation, 16th Triennial Meeting Lisbon, Paris: ICOM; 2011

\section{Submit your manuscript to a SpringerOpen ${ }^{\circ}$ journal and benefit from:}

- Convenient online submission

- Rigorous peer review

- Immediate publication on acceptance

- Open access: articles freely available online

- High visibility within the field

- Retaining the copyright to your article 